\title{
POLÍTICA DE COMPETITIVIDADE E DESENVOLVIMENTO: UMA ANÁLISE DA MATURIDADE DA GESTÃO DE INOVAÇÃO DE EMPRESAS DO SUL DA BAHIA
}

\section{COMPETITIVENESS AND DEVELOPMENT POLICY: A MATURITY ANALYSIS OF BUSINESS INNOVATION MANAGEMENT IN SOUTHERN BAHIA}

\author{
Leidiane Alcântara Brandão ${ }^{1}$; Andréa da Silva Gomes ${ }^{2}$; Gesil Sampaio Amarante Segundo ${ }^{3}$ \\ ${ }^{1}$ Universidade Estadual de Santa Cruz (UESC) - Ilhéus - BA - Brasil \\ brandao.leidiane@gmail.com \\ ${ }^{2}$ Universidade Estadual de Santa Cruz (UESC) - Ilhéus - BA - Brasil \\ andreauesc@gmail.com \\ ${ }^{3}$ Universidade Estadual de Santa Cruz (UESC) - Ilhéus - BA - Brasil \\ gesil.amarante@gmail.com
}

\section{Resumo}

Embutido na lógica de políticas públicas de aumento de competitividade e, desenvolvimento empresarial que visam elevar a qualidade e percepção de valor dos produtos e serviços nacionais, projetos de apoio à atividade de inovação vem sendo estruturados e levados ao mercado nos últimos anos, tornando-se importante identificar as características e desempenho em inovação das empresas envolvidas. Desta forma, este artigo ocupa-se em delinear o comportamento de empresas, localizadas no Sul da Bahia, quanto à gestão da inovação a partir de um método experimental que incluiu aplicação dos indicadores da Matriz JOIN de inovação (2013) e, realização de uma análise comparativa entre um grupo experimental, caracterizado por contratantes do projeto de inovação JOIN e, um grupo de empresas que possuem características semelhantes, mas que não participam do projeto - denominado empresas Gêmeas (grupo de controle). Os resultados indicam que as empresas JOIN possuem desempenho superior na maturidade na atividade de inovação, confirmando a hipótese de que as empresas que aderem a projetos de inovação tendem a apresentar características diferenciadas, que lhes conferem maiores competências para inovar.

Palavras-chave: Diagnóstico; Maturidade; Inovação; Jogo da inovação.

\section{Introdução}

É imprudente dizer que a inovação é preponderante a todas as empresas, pois não há consenso sobre este assunto, mas é inegável que esta tem sido uma palavra de ordem tanto no meio acadêmico quanto nas mídias e, vem orientando diversos direcionamentos ao setor empresarial. De modo contundente, compõe a agenda de políticas públicas de incentivo ao desenvolvimento econômico, com destaque para iniciativas que vêm sendo colocadas em prática no sentido de 
promover a difusão da inovação, incluindo neste processo, uma série de instrumentos de apoio à inovação no setor produtivo, por meio de projetos articuladores de capacitação em gestão da inovação.

Neste contexto, insere-se o JOIN (Jogo da Inovação), uma tecnologia de gestão para sistematizar a atividade de inovação empresarial que se reveste de grande importância, pois ancorado na abordagem de Sistema Nacional de Inovação, representa uma iniciativa do poder público brasileiro para promoção e difusão da cultura inovadora. Destacando-se como fruto do Programa Nacional de Sensibilização e Mobilização para a Inovação, por meio da chamada pública MCT/FINEP - AT - Pró-Inova - Núcleos de Apoio à Gestão da Inovação - 11/2010 que viabilizou a implantação de 24 projetos de inovação com NAGIs (Núcleo de Apoio à Gestão da Inovação) em 12 estados, sendo o JOIN o único projeto NAGI da Bahia (ZEN et al., 2014).

As bases destas iniciativas, de estímulo à competitividade empresarial, remontam-se à década de 1990 com os temas de gestão da qualidade e que mais recentemente, na última década, veio acompanhada por uma Política Industrial (BRANDÃO e DRUMOND, 2012), articulada pela PITCE (Política Industrial, Tecnológica e de Comércio Exterior) em 2004 e PDP (Política de Desenvolvimento Produtivo) em 2008, fazendo parte de um esforço particularmente substancial da política industrial do Plano Brasil Maior (2011-2014).

No âmbito nacional, duas leis se destacam no sentido de construção de parcerias públicoprivadas para fomento da competitividade, com foco em inovação tecnológica: A Lei de Inovação (10.973/2004), voltada para as ICTs (Instituições de Ciência e Tecnologia), das quais exige criação de NITs (Núcleos de Inovação Tecnológica), agências que se articulam com o setor privado por meio de apoio e transferência tecnológica. A outra é a Lei 11.196/2005, conhecida como Lei do bem, que é diretamente voltada para o setor privado, garantindo isenção fiscal a empresas que possuem atividades de inovação tecnológica.

Buscando contribuir com a avaliação de política pública voltada ao fomento da inovação empresarial, esta pesquisa debruça-se em identificar o nível de maturidade da atividade de inovação das organizações atraídas pelo projeto de inovação JOIN, bem como identificar padrões que as diferenciam de empresas com perfil e características semelhantes. Porém, para subsidiar o entendimento do escopo e funcionamento do projeto JOIN (que ainda conta com poucas publicações), parte-se da sua descrição, fundamentada no acesso ao conteúdo didático (Caderno JOIN), consultas ao ambiente digital (Portal JOIN) e, informações verbais transmitidas pela equipe do referido projeto.

\section{Caracterização do JOIN}


No contexto em que é identificado nesta pesquisa é possível observar diversos usos para o termo "JOIN", confirmando a ele um caráter polissêmico, pois denomina: Projeto de inovação, metodologia, tecnologia de gestão, uma filosofia operacional e, até mesmo, a equipe de pesquisadores que o desenvolveu. Como ponto de partida o termo ocupou-se de representar o projeto NAGI do IEL-Bahia, porém, passou a incluir o sentido de metodologia, denominando-se "metodologia JOIN" o uso de ferramentas visuais, dinâmicas e lúdicas, associadas a um processo com fases bem definidas e articuladas que persegue a sistematização da atividade de inovação. Inserido nesta trajetória de evolução e acúmulo de significados, o JOIN adquiriu personalidade de inovação organizacional e passou a ser conhecido, também, como uma tecnologia de gestão. O termo foi estendido, ainda, a uma filosofia operacional, consistindo em um modo de condução da atividade de inovação orientada pela cultura de colaboração, com caráter dialógico e proativo.

O emprego do termo JOIN, para os fins desta pesquisa, centra-se prioritariamente na personalidade de projeto, o qual, por vezes, funde-se às suas características anteriormente mencionadas.

O projeto JOIN busca implantar nas empresas brasileiras um modo de gestão da inovação centrado em atividades colaborativas, dinâmicas e com linguagem lúdica, inserindo a atividade de inovação na rotina empresarial, e para isso utiliza ferramentas de gestão que facilitam a comunicação entre atores internos e externos, na busca de soluções para os problemas enfrentados no âmbito da empresa e necessidades do mercado (JOIN, 2013).

O projeto foi implementado, em caráter experimental, nos municípios baianos de Feira de Santana, Ilhéus, Itabuna, Salvador e Região Metropolitana nos anos de 2013 e 2014, por meio do seu conjunto de soluções, que segundo o Portal JOIN (2013) consiste em:

- Partida JOIN - Jogo empresarial, presencial, que auxilia na resolução de problemas e atendimento de demandas, de modo inovador;

- Caderno JOIN - Reúne boas práticas, ferramentas de gestão e jogos empresariais, oferecendo conteúdo de inovação para empresa de modo dinâmico e sistêmico;

- Oficinas de Inovação JOIN - Encontro dos gestores das empresas participantes do JOIN, articulado para o desenvolvimento de competências em gestão da inovação, geração e compartilhamento de ideias, construção de parcerias e estruturação de networking;

- JOIN na empresa - Assessoria semanal, com foco na condução da atividades de inovação na empresa;

- SisJOIN: Sistema online que auxilia a gestão da inovação, estimula a colaboração e envolve todos os funcionários da empresa no desenvolvimento de ideias e implementação de projetos inovadores; 
- Portal JOIN - Portal online que reúne desde diagnóstico gratuito sobre a atividade de inovação (Matriz JOIN), a conteúdos diversos que auxiliam no desempenho da atividade de inovação, disponível no endereço eletrônico: http://www.jogodainovacao.com.br/portal.

Após maturação experimentada durante a vigência do apoio da FINEP e a eficácia dos resultados obtidos, a tecnologia JOIN, passou a ser demandada por outras unidades federativas (Sistema FIEB, 2014), iniciando a transferência tecnológica pelo estado de Pernambuco ao NAGI/PE, coordenados pelo IEL-PE e Porto Digital, ambas as instituições contempladas pelo mesmo edital que o JOIN (FINEP, 2011).

A tecnologia de gestão JOIN teve sua metodologia desenvolvida e coordenada pelo IEL-BA (Instituto Euvaldo Lodi - Bahia), sendo que seus pilares conceituais estão em Schumpeter (1942), no qual a inovação consiste na combinação de recursos, de modo não convencional para criação de algo novo, ou significativamente melhorado, bem como do Manual de Oslo (2005, p. 55), no qual a inovação consiste na "implantação de um produto (bem ou serviço) novo ou significativamente melhorado, ou um processo, ou um novo método de marketing, ou um novo método organizacional nas práticas de negócios, na organização do local de trabalho ou nas relações externas". Ampara-se ainda nas contribuições de Chesbrough (2003), que apresenta a modelagem de inovação aberta, uma abordagem de negócios em que a empresa passa a se articular com atores diversos que estão dispersos no ambiente externo às organizações para formatar inovações de modo colaborativo.

\section{Metodologia JOIN}

A abordagem é realizada por meio de conteúdos atuais, específicos da área de inovação ou adaptados à gestão da inovação, e são amplamente discutidos na literatura especializada (VIANNA, 2012; McAFEE, 2010; CHESBROUGH, 2006; NONAKA e TAKEUCHI, 2008; TIGRE, 2006; MANUAL DE OSLO, 2005), introduzidas pela linguagem lúdica do futebol. O conteúdo abordado (Quadro 1) conecta-se com os indicadores da Matriz JOIN (Quadro 3), utilizado no diagnóstico de identificação do nível de maturidade empresarial, conforme JOIN (2013):

Quadro 1 - Abordagem e conteúdo dos indicadores utilizados na metodologia JOIN.

\begin{tabular}{|c|l|}
\hline Abordagem & \multicolumn{1}{c|}{ Conteúdo } \\
\hline Rede & $\begin{array}{l}\text { Dedica-se aos pilares da inovação empresarial, incluindo diferentes modelos de gestão da inovação } \\
\text { como inovação aberta, redes de inovação, alinhamento entre inovação e sustentabilidade, bem como os } \\
\text { sistemas de inovação. }\end{array}$ \\
\hline Arena & $\begin{array}{l}\text { Espaço dedicado à gestão de conhecimento na empresa e à promoção da inteligência do negócio por } \\
\text { meio da estruturação de ambientes físicos e digitais que dinamizam a comunicação e a colaboração na } \\
\text { atividade de inovação. }\end{array}$ \\
\hline Regras & $\begin{array}{l}\text { Espaço dedicado à construção da agenda de inovação da empresa: definição de estratégias, modelos de } \\
\text { negócio e políticas que facilitam a realização do propósito, dos objetivos e metas da organização pela } \\
\text { via da inovação. }\end{array}$ \\
\hline Time & Espaço dedicado à gestão do desenvolvimento e do engajamento das pessoas na atividade de inovação. \\
\hline
\end{tabular}




\begin{tabular}{|c|l|} 
Jogo & $\begin{array}{l}\text { Espaço dedicado ao processo de inovação: o conjunto de atividades articuladas e interdependentes que } \\
\text { aceleram o desenvolvimento e a introdução de inovações no mercado. }\end{array}$ \\
\hline Gol & $\begin{array}{l}\text { Espaço da apuração das ofertas de inovação, indicando quantas inovaços a empresa introduziu no } \\
\text { mercado nos últimos dois anos. }\end{array}$ \\
\hline
\end{tabular}

Fonte: Adaptado de JOIN (2013)

A proposta do JOIN ofertada na Região Sul da Bahia contemplou a implementação de sua solução em dois momentos complementares: Capacitação de gestores (64hs) e visitas técnicas (64hs). No modelo de oficinas de inovação, o treinamento, conduzido por técnicos $\mathrm{JOIN}^{1}$, contempla a capacitações de dois gestores por empresa, oportunidade em que empresários de diversos ramos de atividade juntam-se para interagirem com o compartilhamento de experiências, ideias, aprendizados e são apresentados a outros atores e ambientes de cooperação externos, como ambientes virtuais de articulação em rede, instituições e agentes sociais que apresentam soluções coletivas para problemas diversos. As visitas técnicas, disponibilizadas em 16 assessorias, são conduzidas por um técnico JOIN, que auxilia um grupo de cinco a sete pessoas a construir as bases para a sistematização da atividade de inovação na empresa, personalizando a abordagem ao nível de maturidade, porte, disponibilidade financeira e capacidade de captação de subsídios econômicos, tecnológicos e informacionais.

De modo experimental, adaptado às demandas e peculiaridades da região Sul da Bahia, foi desenvolvido e testado em uma das empresas um modelo de implementação da metodologia JOIN $100 \%$ in company. Este modelo fundiu capacitações e assessorias em 64 horas e os principais ganhos desta adaptação, segundo percepção dos empresários e do técnico JOIN, consistiu na personalização da abordagem à empresa e mais colaboradores capacitados nos temas da oficina, além de redução de custo operacional.

Em ambos os modelos de implementação, o resultado esperado foi que as empresas coloquem em prática os recursos acessados com o projeto, e com isso, elevem sistematicamente o nível de maturidade identificada no diagnóstico preliminar, promovendo melhor gestão e oferecendo inovações de maior valor agregado.

\subsection{Empresas atraídas ao JOIN: Critérios de seleção}

No esforço em atrair parte das empresas baianas para a implementação do JOIN e, sensibilizá-las para os temas de inovação, foram realizadas diversas ações para engajamento ao projeto, envolvendo eventos de inovação, chamadas televisivas e em mídias digitais, bem como envio de convites e visitas às empresas. No entanto, apesar da ampla convocação empresarial, a estatística de empresas que se empenharam em aderir foi abaixo da capacidade de atendimento.

\footnotetext{
${ }^{1}$ Os técnicos JOIN são bolsistas CNPQ que participaram desde a concepção e desenvolvimento da metodologia, à fase de implementação nas empresas. O JOIN foi concebido de forma multidisciplinar, de modo que contou com 18 pesquisadores bolsistas de diversas áreas de conhecimento.
} 
Controles internos do projeto JOIN (informação verbal ${ }^{2}$ ), indicam que no período de Novembro de 2012 a Agosto de 2014, há registro de 415 empresas sensibilizadas ${ }^{3}: 88$ empresas em Feira de Santana, 214 em Salvador e região metropolitana, e 113 na região Sul da Bahia (Ilhéus e Itabuna). Todavia, apenas 41 empresas em todo estado da Bahia aderiram ao projeto, o que representa apenas $10 \%$ daquelas que foram sensibilizadas. Esse fato chama a atenção, pois as empresas que aderiram ao JOIN possivelmente possuem características diferenciadoras das demais, pois reagiram de modo distinto a um mesmo estímulo que foi dado a todas as empresas apresentadas ao projeto.

Mais especificamente para região Sul da Bahia, entre todos os contatos realizados e das 113 empresas que foram pessoalmente apresentadas ao JOIN, apenas 8 empresas (7\%) aderiram ao projeto, prevendo alocação de tempo e dinheiro para sistematização da atividade de inovação. Desta forma, a quantidade de adesão ao projeto situando-se abaixo do esperado, inviabilizou a adoção de critérios mais rigorosos de seleção das empresas participantes, pois não foi possível efetivar priorização com base em níveis iniciais de maturidade da atividade de inovação, contou-se com o comportamento pró-inovação, materializado pelo interesse em aderir ao projeto.

\section{Metodologia de pesquisa}

A pesquisa exploratória foi composta de aplicação de questionários orientados por indicadores de inovação, aplicados aos gestores de uma amostra multisetorial de 15 empresas localizadas na região Sul da Bahia, representadas por códigos alfanuméricos e, subdivididas em dois grupos:

- Um grupo experimental constituído por oito empresas que assinaram contrato, confirmando interesse em implementar o projeto de inovação JOIN nos anos de 2013 e 2014, denominadas empresas JOIN;

- Sete empresas do grupo de controle, que embora possuam características semelhantes às empresas JOIN, diferenciam-se, inicialmente, por não aderirem ao projeto de inovação.

Deste modo, o modelo empírico empregado buscou identificar padrões e comportamentos relacionados à inovação que as distinguem. Adotou-se como método de pesquisa a realização de uma análise comparativa entre estas empresas, cujo estudo desdobra-se em duas etapas:

a) Caracterização do perfil das empresas, destacando dados como: Porte ${ }^{4}$, número de funcionários e escolaridade dos gestores;

\footnotetext{
${ }^{2}$ Informação fornecida pela equipe JOIN em outubro de 2014, com base em relatórios de acompanhamentos internos do IEL-BA.

${ }^{3}$ Considera-se empresas sensibilizadas aquelas que participaram de algum evento ou recebeu visita de membros da equipe JOIN para sensibilizá-los para o tema de inovação e apresentar pessoalmente a proposta do projeto.

4 O porte empresarial foi baseado no enquadramento da lei $\mathrm{N}^{\mathrm{o}} 123$, de 14 de dezembro de 2006 que institui o Estatuto Nacional da Microempresa e da Empresa de Pequeno Porte, pois apesar do JOIN utilizar a classificação do BNDES,
} 
b) Identificação do Índice de Maturidade da Atividade de Inovação (IMAI) adaptado de Matriz JOIN (2013), também conhecido como radar da inovação. Assenta-se em indicadores agrupados em seis abordagens: Rede, Arena, Regras, Time, Jogo (capacidade inovativa) e Gol (ofertas de inovação), Quadro 3.

Para identificação das capacidades inovativas os gestores foram questionados: "Como as atividades/procedimentos apresentados pelos indicadores de inovação apresentam-se na sua empresa?”. Destacando-se a legenda (Quadro 1) como parâmetro de respostas. Enquanto isto, para identificação do índice da oferta de inovação, questionou-se: "Quantas inovações sua empresa introduziu no mercado nos últimos dois anos?". Para avaliar os resultados é utilizada uma escala numérica $(0,1,2,3$ ou, 4 ou mais inovações).

Quadro 2 - Legenda para identificação do nível de maturidade da atividade de inovação, referente aos indicadores da Matriz JOIN (2013)

\begin{tabular}{|c|l|}
\hline \multicolumn{1}{|c|}{ GRAU } & \multicolumn{1}{c|}{ CARACTERÍSTICAS } \\
\hline $1-\quad$ Inexistente & Não acontece na empresa. \\
\hline 2- $\quad$ Informal & $\begin{array}{l}\text { Acontece de forma não estruturada, sem rigor no planejamento ou na execução. A } \\
\text { solução emerge ao acaso, de forma aleatória, e baseia-se na percepção das lideranças. }\end{array}$ \\
\hline 3- Estruturado & $\begin{array}{l}\text { Acontece de forma estruturada, usando, ainda que de forma parcial, procedimentos } \\
\text { padronizados e instrumentos formais de controle. }\end{array}$ \\
\hline 4- Sistematizado & $\begin{array}{l}\text { Procedimento totalmente difundido na organização e incorporado à rotina } \\
\text { organizacional, controlado com o uso eficiente de sistemas de monitoramento e } \\
\text { avaliação. }\end{array}$ \\
\hline 5- Otimizando & Procedimento incorporado e em processo contínuo de revisão e melhoria. \\
\hline
\end{tabular}

Fonte: Adaptado de JOIN (2013)

Quadro 3 - Abordagens e Indicadores relativos à Maturidade da Atividade de Inovação

\begin{tabular}{|c|c|c|c|}
\hline \multirow{5}{*}{$\sum_{\substack{\infty \\
0}}^{\infty}$} & \multirow{5}{*}{ : } & 串 & $\begin{array}{l}\text { A Atividade de Inovação da Empresa: Alocação de tempo e recursos no desenvolvimento de } \\
\text { produtos, serviços e processos novos ou significativamente melhorados; Alocação de tempo e recursos } \\
\text { no desenvolvimento de novas soluções de marketing e gestão; Integração da força de trabalho no } \\
\text { esforço de inovação; Integração de atores externos - outras empresas, Instituiços Científicas e } \\
\text { Tecnológicas, especialistas e consultores - no esforço de inovação; Integração de consumidores no } \\
\text { esforço de inovação; Investimento na capacitação da força de trabalho, para o desenvolvimento de } \\
\text { competências técnicas e comportamentais; Participação em redes de inovação; Atividade de Pesquisa e } \\
\text { Desenvolvimento. }\end{array}$ \\
\hline & & \multirow{3}{*}{ 胥 } & $\begin{array}{l}\text { Empresa 2.0: Integração de ferramentas e sistemas da web } 2.0 \text { disponíveis na Internet na atividade de } \\
\text { inovação; Uso de um sistema computacional específico de comunicação e colaboração - Software. }\end{array}$ \\
\hline & & & $\begin{array}{l}\text { Gestão do Conhecimento: Iniciativas que estimulam a colaboração intraorganizacional; Iniciativas que } \\
\text { facilitam o compartilhamento de boas práticas, informações e conhecimento na empresa; Iniciativas que } \\
\text { facilitam a captura e o desenvolvimento de ideias para inovações na empresa; Iniciativas que facilitam a } \\
\text { tomada conjunta de decisões. }\end{array}$ \\
\hline & & & $\begin{array}{l}\text { Inteligência Competitiva: Monitoramento da dinâmica do mercado; Monitoramento de iniciativas } \\
\text { públicas e privadas de estímulo à inovação; Monitoramento de fontes de informações críticas para o } \\
\text { negócio; Participação em eventos importantes para o negócio; Participação em entidades associativas } \\
\text { industriais; Tratamento dos dados coletados sobre o ambiente externo para a identificação de } \\
\text { oportunidades de inovação; Tratamento dos dados internos para a identificação de padrões e } \\
\text { oportunidades de inovação; Aplicação, no negócio, das informações obtidas no esforço de inteligência } \\
\text { competitiva; Atualização sobre novos conhecimentos de base científico-tecnológica. }\end{array}$ \\
\hline & & & Estratégia de inovação: Construção da estratégia de inovação da empresa; Atualização da estratégia de \\
\hline
\end{tabular}

observou-se que esta classificação apresenta-se mais aderente às empresas relacionadas nesta pesquisa, devido à lacuna de faturamento entre as duas classificações. 


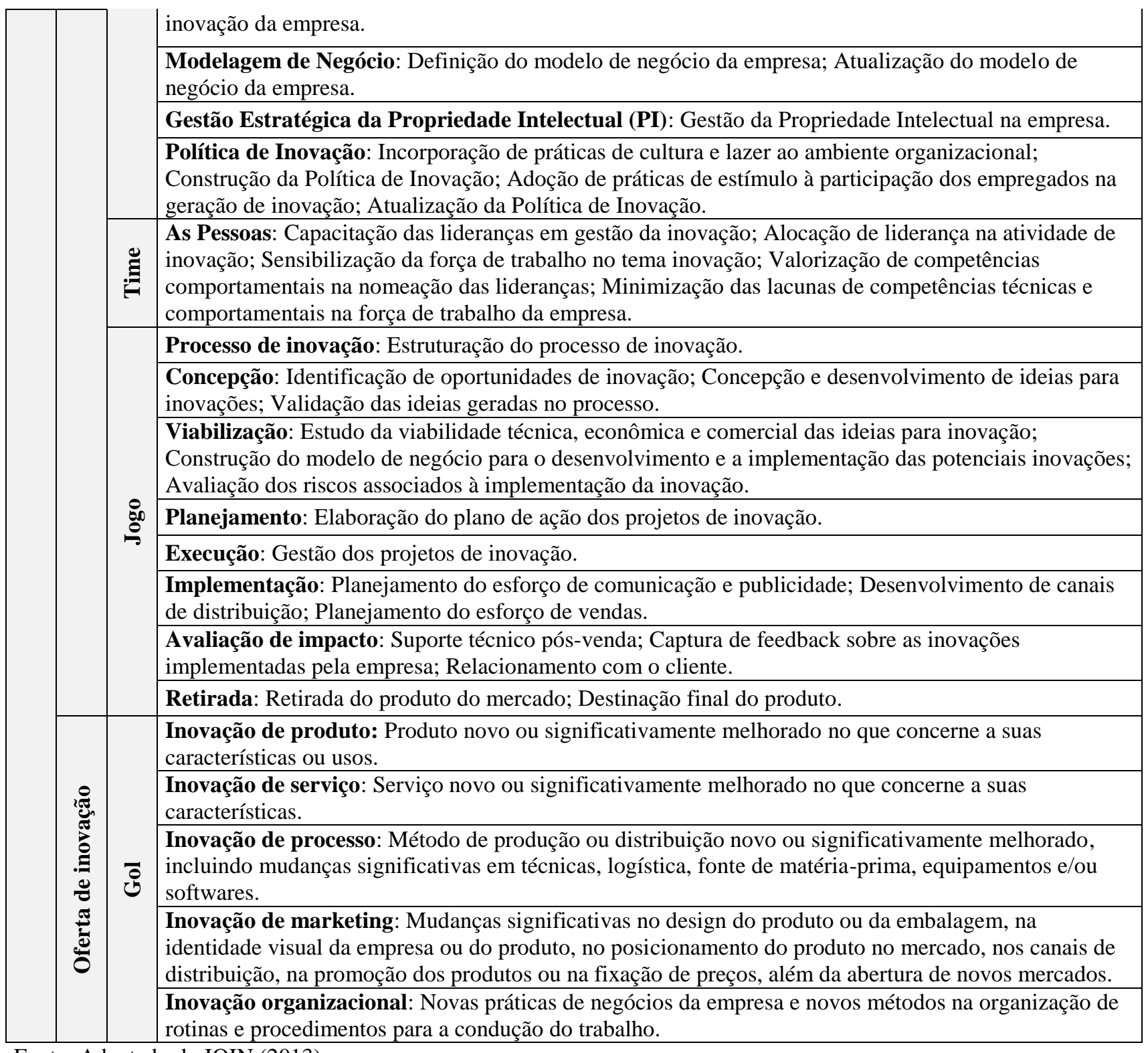

Fonte: Adaptado de JOIN (2013)

O IMAI de cada abordagem pode ser calculado por meio da equação a seguir:

$$
\mathrm{IMAI}=\frac{5}{5 \mathrm{n}} \mathrm{S}
$$

Onde: $\left(\mathrm{n}=\mathrm{n}^{\mathrm{o}}\right.$ de indicadores da temática; $\mathrm{S}=$ somatório da escala).

Considera-se a seguintes quantidades de indicadores por temática: $\operatorname{Rede}=8$; Arena $=15$; Regras $=9 ;$ Time $=5 ;$ Jogo $=17 ; \mathrm{Gol}=5$, ao tempo em que se convenciona o somatório da escala (S) como cumulativo, ou seja, cada possibilidade de marcação equivale a um ponto por indicador.

Para mensurar o IMAI Global (por empresa), calcula-se:

IMAI Global $=\frac{\sum \text { IMAI }}{6}$ 
As características de cada IMAI são as mesmas identificadas na legenda do quadro 2, ou seja, varia de inexistente a otimizando.

\section{Análise e discussão dos resultados}

Os resultados da pesquisa de campo e as discussões detalham as características das amostras estudadas, realizando-se uma análise comparativa fundamentada por estatística descritiva, apresentados por índices, gráficos, quadros e tabelas.

\subsection{Caracterização do perfil das empresas JOIN}

Entre as características comuns do conjunto de empresas JOIN, sumarizadas na tabela 1, a média de 23 anos no mercado chama a atenção pelo nível de maturidade empresarial, quanto ao grau de resiliência. Apenas uma empresa tem menos de 3 anos de sobrevivência, período de maior risco de mortalizada para as MPE, de modo que, neste sentido, as demais empresas encontram-se consolidadas no mercado, sinalizando acúmulo de aprendizado com a atividade empresarial para competir. Quanto à empresa com apenas 2 anos de existência é importante destacar que trata-se de uma spin off, ou seja, surgiu de outra empresa do mesmo ramo de atividade como oportunidade de negócio complementar à atividade já desenvolvida, dessa forma a empresa usufrui de aprendizado e conhecimento de 24 anos da empresa "mãe", elevando consideravelmente sua maturidade em termos de experiência de mercado e compartilhando capital humano.

O aprendizado organizacional pode ser mensurado pela prática desenvolvida ao longo de sua existência, no entanto a educação formal tem papel importante na condução das atividades, deste modo a escolaridade do gestor pode trazer reflexos importantes para sua maturidade em inovação. Enquanto que a quantidade de funcionários oscila de acordo com o setor de atividade e o porte da empresa, podendo ter relação direta com a capacidade de difusão da inovação na empresa.

Tabela 1 - Características gerais sobre o perfil das empresas JOIN, localizadas na Região Sul da Bahia (2013-

\begin{tabular}{|c|c|c|c|c|c|c|c|}
\hline \multicolumn{8}{|c|}{ 2014) } \\
\hline Empresa & $\begin{array}{l}\text { Anos no } \\
\text { mercado }\end{array}$ & $\begin{array}{c}\mathrm{N}^{\mathbf{0}} \text { de } \\
\text { Funcionários }\end{array}$ & $\begin{array}{c}\text { Escolaridade do } \\
\text { gestor (Concluída) }\end{array}$ & $\begin{array}{l}\text { Setor de atividade } \\
\text { (Principal) }\end{array}$ & Porte & Atividade & Município \\
\hline A & 20 & 11 & $2^{\circ} \mathrm{Grau}$ & $\begin{array}{c}\text { Produtos de } \\
\text { limpeza } \\
\text { Embalagens de }\end{array}$ & EPP & $\begin{array}{l}\text { Indústria/ } \\
\text { Comércio }\end{array}$ & Ilhéus \\
\hline B & 47 & 13 & Especialista & $\begin{array}{c}\text { papel } \\
\text { Lanchonete e }\end{array}$ & EPP & $\begin{array}{l}\text { Indústria } \\
\text { Indústria/ }\end{array}$ & Ilhéus \\
\hline $\mathrm{C}$ & 18 & 30 & $3^{\circ} \mathrm{Grau}$ & confeitaria & EPP & Comércio & Itabuna \\
\hline $\mathrm{D}$ & 29 & 38 & $3^{\circ} \mathrm{Grau}$ & $\begin{array}{c}\text { Derivados do cacau } \\
\text { e chocolate } \\
\text { Produção de }\end{array}$ & EPP & $\begin{array}{l}\text { Indústria/ } \\
\text { Comércio } \\
\text { Indústria/ }\end{array}$ & Ilhéus \\
\hline $\mathrm{E}$ & 2 & 8 & $3^{\circ} \mathrm{Grau}$ & $\begin{array}{l}\text { Argamassas } \\
\text { Lanchonetes e }\end{array}$ & $\mathrm{ME}$ & $\begin{array}{l}\text { Comércio } \\
\text { Indústria/ }\end{array}$ & Ilhéus \\
\hline $\mathrm{F}$ & 30 & 21 & $1^{\circ} \mathrm{Grau}$ & restaurante & EPP & Comércio & Ilhéus \\
\hline $\mathrm{G}$ & 23 & 6 & $2^{\circ} \mathrm{Grau}$ & Serviços gráficos & $\mathrm{ME}$ & Serviços & Ilhéus \\
\hline
\end{tabular}


$\mathrm{H}$

15

15

$2^{\circ} \mathrm{Grau}$

Supermercado

EPP Comércio

Ilhéus

Nota: EPP (Empresa de Pequeno Porte); ME (Microempresa).

Fonte: Pesquisa de campo (2015). 


\subsection{Caracterização do Perfil das Empresas Gêmeas}

As empresas que se configuram como Gêmeas, que metodologicamente representam um grupo de controle, foram selecionadas por possuírem características muito similares quanto à atividade desenvolvida e atuarem no mesmo mercado que as respectivas empresas JOIN. Adicionalmente, elas foram consideradas potenciais clientes JOIN, possuindo os requisitos para adesão ao projeto.

Para além dos atributos de classificação como empresas Gêmeas, o resultado da pesquisa sobre características gerais revelou que uma das diferenças centrais entre estas empresas e as JOIN incide no tempo de atuação no mercado (Tabela 2). A média de sobrevivência das Gêmeas é de oito anos, tempo de experiência de mercado relativamente baixo em analogia às empresas JOIN, pois são quase 3 três vezes mais jovens, em contrapartida, a média de escolaridade é levemente mais elevada. Estas duas variáveis (sobrevivência e escolaridade) podem estar correlacionadas, tendo em vista que gestores mais jovens ou com atividade empresarial mais recente começaram suas atividades em um ambiente mais competitivo que exige um maior nível de educação formal, além da ampliação do acesso à educação nos últimos anos.

Tabela 2 - Características gerais sobre o perfil das empresas Gêmeas** localizadas na Região Sul da Bahia (2014)

\begin{tabular}{|c|c|c|c|c|c|c|c|}
\hline \multicolumn{8}{|c|}{ Caracterização das empresas GÊEEAS } \\
\hline Empresa & $\begin{array}{l}\text { Anos no } \\
\text { mercado }\end{array}$ & $\begin{array}{c}\mathrm{N}^{\circ} \text { de } \\
\text { Funcionários }\end{array}$ & $\begin{array}{c}\text { Escolaridade } \\
\text { do gestor } \\
\text { (Concluída) } \\
\end{array}$ & $\begin{array}{l}\text { Setor de atividade } \\
\text { (Principal) }\end{array}$ & Porte & Atividade & Município \\
\hline A1 & 12 & 4 & $3^{\circ} \mathrm{Grau}$ & Produtos de limpeza & $\mathrm{ME}$ & Comércio & Ilhéus \\
\hline B1 & 12 & 22 & $2^{\circ} \mathrm{Grau}$ & Embalagens de papel & EPP & Indústria & Ilhéus \\
\hline $\mathrm{C} 1$ & 2 & 10 & $3^{\circ} \mathrm{Grau}$ & $\begin{array}{l}\text { Lanchonete e } \\
\text { confeitaria }\end{array}$ & $\mathrm{ME}$ & $\begin{array}{l}\text { Indústria/ } \\
\text { Comércio }\end{array}$ & Itabuna \\
\hline D1 & $*$ & $*$ & $*$ & $*$ & $*$ & $*$ & $*$ \\
\hline E1 & 3 & 14 & Especialista & $\begin{array}{c}\text { Produção de } \\
\text { Argamassas } \\
\text { Lanchonetes e }\end{array}$ & $\mathrm{ME}$ & $\begin{array}{l}\text { Indústria } \\
\text { Indústria/ }\end{array}$ & Ilhéus \\
\hline $\mathrm{F} 1$ & 23 & 28 & $2^{\circ} \mathrm{Grau}$ & restaurante & EPP & Comércio & Ilhéus \\
\hline G1 & 4 & 6 & $3^{\circ} \mathrm{Grau}$ & Serviços gráficos & $\mathrm{ME}$ & Serviços & Ilhéus \\
\hline H1 & 5 & 5 & $3^{\circ} \mathrm{Grau}$ & Supermercado & EPP & Comércio & Ilhéus \\
\hline
\end{tabular}

Nota: EPP (Empresa de Pequeno Porte); ME (Microempresa); (*) Não houve coleta de dados para a gêmea D1 - As empresas que se configuram como Gêmeas deste setor optaram por não participar da pesquisa. (**) Empresas com perfil e setor de atividade semelhante às empresas inseridas no JOIN (2013-2014) na Região Sul da Bahia.

Fonte: Pesquisa de campo (2015).

\subsection{Nível de Maturidade da Atividade de Inovação: Empresas JOIN x Empresas Gêmeas}

Uma análise pareada entre os dois grupos de empresas, permite visualizar um comportamento semelhante no que se refere ao nível de maturidade de ambos, em algumas abordagens específicas, observando-se uma tendência de destaque entre os indicadores relativos a “concepção", "modelagem de negócio", e "oferta de inovação" em detrimento a "empresa 2.0". 
Este comportamento, possivelmente, está relacionado à cultura empresarial que estão inseridas, bem como pode ser característico da competitividade dos setores analisados na área de estudo (Figura 1).

Figura 1 - Índice de Maturidade da Atividade de Inovação (IMAI) das empresas JOIN e empresas Gêmeas* localizadas na Região Sul da Bahia distribuído por indicadores (2013-2014)

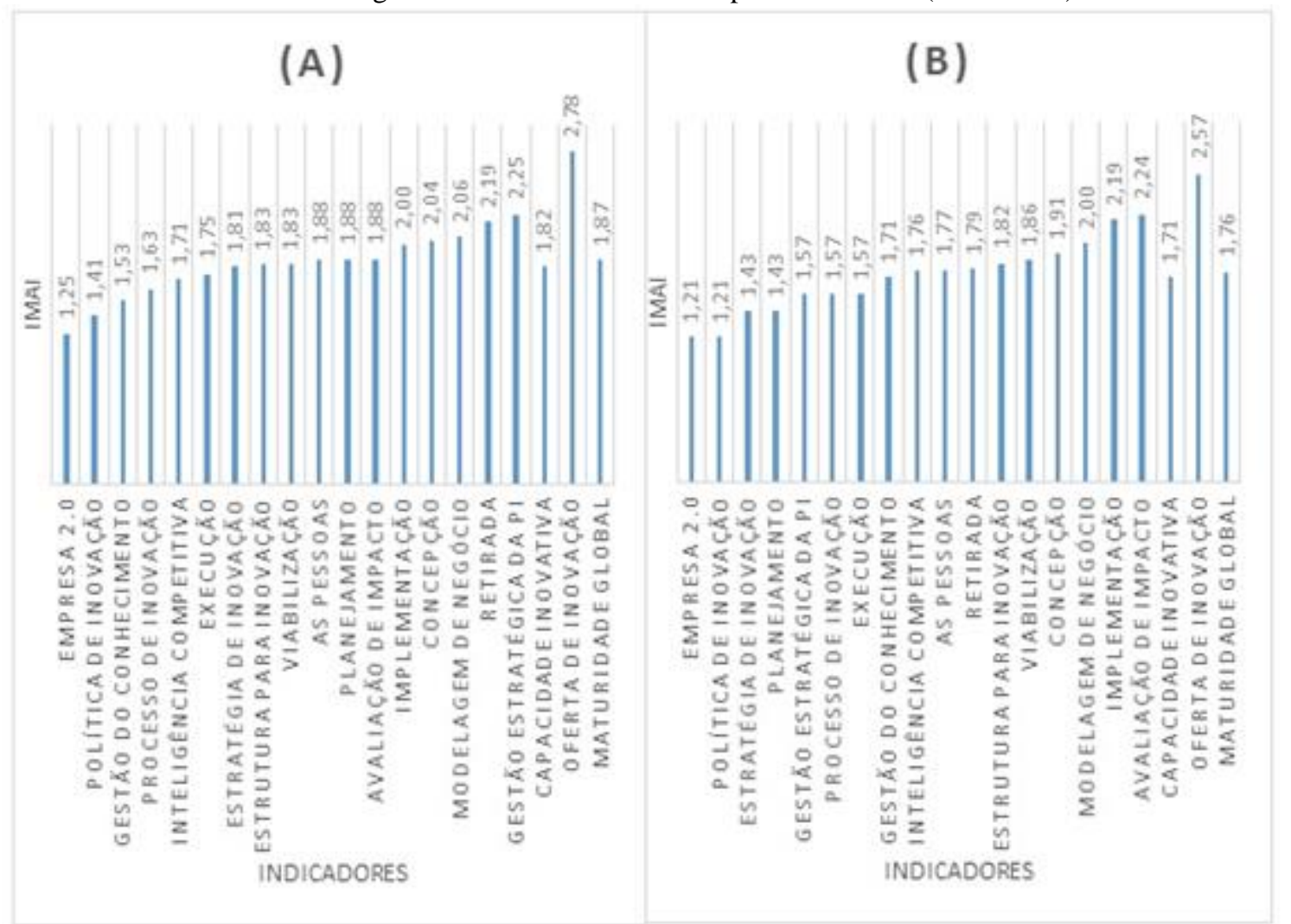

Nota: (A) Empresas JOIN; (B) Empresas Gêmeas; IMAI: Índice de Maturidade da Atividade de inovação. (*) Empresas com perfil e setor de atividade semelhante às empresas inseridas no JOIN (2013-2014) na Região Sul da Bahia.

Fonte: Pesquisa de campo (2015).

Dentro desta perspectiva, a média dos IMAIs (Índices de Maturidade da Atividade de Inovação) das empresas JOIN e Gêmeas coincidem-se ao manter no limite inferior a temática “empresa 2.0", que diz respeito ao uso de plataformas digitais de colaboração, e no limite superior “oferta de inovação". As empresas JOIN mantem limites 1,25-2,78 e empresas Gêmeas 1,21-2,57, não apresentando uma lacuna expressiva entre os limites inferiores e superiores dos dois grupos que situam entre os níveis inexiste a informal, tendendo a estruturado quanto mais se aproxima de IMAI 3.

Apesar de certa proximidade no nível de maturidade em algumas abordagens, há um comportamento irregular entre os dois grupos de empresas, de modo que as empresas JOIN usufruem de uma leve vantagem na maioria das temáticas investigadas, garantindo uma média global de 0,11 pontos à frente das empresas Gêmeas (Tabela 3 e Tabela 4).

O nível global do IMAI das empresas JOIN (Tabela 3) revela que a sua capacidade nos indicadores investigados equivale a 1,96, expressando que o nível de inovação dessas empresas 
tende ao nível informal, um estágio típico de um ambiente em que as atividades e procedimentos que direcionam à inovação se apresentam de forma não estruturada, sem rigor no planejamento ou na execução, a solução baseia-se na percepção da liderança e emerge aleatoriamente.

Este grau de maturidade, tendendo a informal, é relevante ao se levar em conta a especificidade dos 59 indicadores investigados, pois o nível 1 equivale a inexistente, enquanto o grau 2 sinaliza que os aspectos de inovação são observados na empresa, mesmo que conduzido de modo pessoal pelo gestor. Enquanto isso, o nível global do IMAI das empresas Gêmeas (Tabela 4) é de 1,85 , de modo que também se aproxima mais do nível informal que inexistente, constituindo um indicador relevante apesar de inferior às empresas JOIN.

Tabela 3 - Índice de Maturidade da Atividade de Inovação (IMAI) das empresas JOIN da Região sul da Bahia, distribuído por abordagem (2013-2014)

\begin{tabular}{lccccccccc} 
Abordagem / Empresa JOIN & $\mathbf{A}$ & $\mathbf{B}$ & $\mathbf{C}$ & $\mathbf{D}$ & $\mathbf{E}$ & $\mathbf{F}$ & $\mathbf{G}$ & H & Índice IMAI \\
\hline CAPACIDADE INOVATIVA* & $\mathbf{1 , 9 1}$ & $\mathbf{2 , 3 5}$ & $\mathbf{1 , 9 5}$ & $\mathbf{1 , 6 5}$ & $\mathbf{1 , 6 2}$ & $\mathbf{1 , 9 8}$ & $\mathbf{1 , 4 1}$ & $\mathbf{1 , 5 1}$ & $\mathbf{1 , 8 0}$ \\
\hline Rede & 1,75 & 2,75 & 1,75 & 1,50 & 2,00 & 2,25 & 1,13 & 1,50 & 1,83 \\
Arena & 1,64 & 1,66 & 1,63 & 1,26 & 1,48 & 1,59 & 1,37 & 1,34 & 1,50 \\
Regras & 1,56 & 3,44 & 2,19 & 1,75 & 1,50 & 1,94 & 1,44 & 1,25 & 1,88 \\
Time & 2,60 & 1,60 & 2,00 & 1,80 & 1,60 & 2,00 & 1,60 & 1,80 & 1,88 \\
Jogo & 2,02 & 2,29 & 2,17 & 1,92 & 1,50 & 2,13 & 1,50 & 1,67 & 1,90 \\
Gol & 2,80 & 4,20 & 4,60 & 3,20 & 2,20 & 2,40 & 1,40 & 1,40 & 2,78 \\
\hline Nível Global do IMAI & $\mathbf{2 , 0 6}$ & $\mathbf{2 , 6 6}$ & $\mathbf{2 , 3 9}$ & $\mathbf{1 , 9 1}$ & $\mathbf{1 , 7 1}$ & $\mathbf{2 , 0 5}$ & $\mathbf{1 , 4 1}$ & $\mathbf{1 , 4 9}$ & $\mathbf{1 , 9 6}$ \\
\hline
\end{tabular}

Nota: Setor de atividade: A: Produtos de limpeza; B: Embalagens de papel; C: Lanchonete e confeitaria; D: Produtos derivados do cacau e chocolate; E: Produção de Argamassas; F: Lanchonetes e restaurante; G Serviços gráficos; H: Supermercado. (*) A Capacidade Inovativa equivale à média das abordagens Rede; Arena; Regras; Time; Jogo.

Fonte: Pesquisa de campo (2015).

Tabela 1 - Índice de Maturidade da Atividade de Inovação (IMAI) das empresas Gêmeas** localizadas na Região Sul da Bahia, distribuído por abordagem (2014)

\begin{tabular}{|c|c|c|c|c|c|c|c|c|c|}
\hline Indicadores / Empresa Gêmeas & A1 & B1 & C1 & D1 & E1 & F1 & G1 & H1 & Índice MAI \\
\hline CAPACIDADE INOVATIVA*** & 1,73 & 1,77 & 1,40 & $*$ & 2,05 & $\mathbf{1 , 8 3}$ & $\mathbf{1 , 7 0}$ & 1,46 & 1,71 \\
\hline Arena & 1,51 & 2,24 & 1,23 & $*$ & 1,70 & 1,55 & 1,24 & 1,47 & 1,56 \\
\hline Regras & 1,50 & 1,44 & 1,13 & $*$ & 2,31 & 1,56 & 1,75 & 1,19 & 1,55 \\
\hline Time & 1,80 & 1,60 & 1,40 & $*$ & 1,60 & 2,00 & 2,20 & 1,80 & 1,77 \\
\hline Jogo & 1,71 & 1,56 & 1,38 & $*$ & 2,50 & 2,17 & 1,81 & 1,61 & 1,82 \\
\hline Gol & 1,80 & 4,40 & 2,80 & $*$ & 3,20 & 2,80 & 1,80 & 1,20 & 2,57 \\
\hline NÍVEL GLOBAL DO IMAI & 1,74 & 2,21 & 1,64 & $*$ & 2,24 & 1,99 & 1,72 & 1,42 & $\mathbf{1 , 8 5}$ \\
\hline
\end{tabular}

Nota: Setor de atividade: A1: Produtos de limpeza; B1: Embalagens de papel; C1: Lanchonete e confeitaria; D1(*): Produtos derivados do cacau e chocolate - Não forneceu dados para pesquisa; E1: Produção de Argamassas; F1: Lanchonetes e restaurante; G1: Serviços gráficos; H1: Supermercado. (**) Empresas com perfil e setor de atividade semelhante às empresas inseridas no JOIN (2013-2014) na Região Sul da Bahia. (***) A Capacidade Inovativa equivale à média das abordagens Rede; Arena; Regras; Time; Jogo.

Fonte: Pesquisa de campo (2015).

A estratificação do nível global do IMAI em indicadores da capacidade inovativa e oferta de inovação (Gol) permitiu a identificação de um padrão no comportamento dos indicadores, que tende a apresentar maior desempenho em maturidade na abordagem "Gol". Entre as empresas JOIN identifica-se que o índice das variáveis qualitativas referentes à capacidade inovativa equivale a 1,80 e o índice relacionado às variáveis quantitativas da temática "gol” equivale a 2,78, ou seja, 
tende a 3 que se refere ao nível estruturado, enquanto a capacidade inovativa inclina-se ao nível 2, informal. Para as empresas Gêmeas este padrão se repete, ou seja, o indicador de oferta de inovação $(2,57)$ é mais elevado que a capacidade inovativa $(1,71)$. Este detalhamento esclarece que o índice da temática "Gol" sobressai frente às outras cinco temáticas em quase um grau de diferença, permitindo inferir que a quantidade de inovações implementadas faz alavancar consideravelmente o nível de maturidade global da atividade de inovação.

Uma análise específica sobre a quantidade de inovações implementadas pelas empresas nos últimos dois anos estreita a identificação dos direcionadores de inovação, e nesse sentido, duas tendências se sobressaem: Uma relacionada ao tipo de inovação preponderante e outra relativa ao setor de atividade.

Em empresas com atividade de produção, os indicadores concernentes à temática "Gol" apresentaram resultados expressivamente mais elevados que os indicadores de capacidade inovativas. Assim, estas empresas conseguem colocar quantidades relevantes de inovações no mercado (em produto, serviço, marketing, processo, organizacional e modelo de negócio) mesmo com pouca estruturação de suas atividades, as quais acontecem de modo preponderantemente informal, ou seja, conduzido pessoalmente pelo gestor e sem mecanismos de controle e monitoramento. Quanto aos tipos de inovações, as empresas com atividades de produção alcançaram grau máximo (Otimizando) em inovação em produto, confirmando competências diferenciadoras das empresas do setor de serviços e setor de comércio, as quais geraram menor variedade e quantidade de inovações entre os tipos investigados.

A comparação entre os indicadores da matriz IMAI (Tabela 3) permite visualizar os índices máximos e mínimos, tanto entre as temáticas quando entre as empresas. Assim, na capacidade inovativa das empresas JOIN, o índice mínimo é de 1,50 em Arena e máximo de 1,90 em o Jogo. A empresa B possui o maior IMAI global $(2,66)$, uma empresa de pequeno porte que desenvolve atividade industrial relacionada à produção de embalagens de papel, enquanto o menor IMAI global é apresentado pela empresa $\mathrm{G}(1,41)$, microempresa que desenvolve atividades de serviços gráficos. Estendendo a mesma análise sobre as empresas Gêmeas (Tabela 4), identifica-se que a capacidade inovativa é de 1,55 (Regras) no índice mínimo, e é sustentada por dois índices máximos, Rede e Jogo, ambos 1,82. A empresa E1, que é uma ME (Microempresa) produtora de argamassas, lidera o nível global do IMAI com 2,24, enquanto a empresa H1, uma ME do ramo comércio varejista ocupa o limite inferior com 1,42.

Convém observar que, por apenas 0,01 de diferença entre os IMAI Arena e Regras das empresas Gêmeas, os dois grupos empresariais não compartilham índices máximos e mínimos referente aos indicadores identificados na tabela 3 e tabela 4, pois, as empresas JOIN possuem 
como índice mínimo (Arena) e máximo (Jogo) e as empresas Gêmeas índice mínimo (Regras) e máximo (Jogo).

De acordo com os limites inferiores da capacidade inovativa, as empresas da Região Sul da Bahia partilham de deficiências semelhantes quanto a apropriação de conteúdos de inovação embutidos na Matriz JOIN, como "gestão de conhecimento" e "inteligência do negócio", que equivalem à estruturação de ambientes que favoreçam a comunicação e colaboração, podendo ser classificados entre informal e inexistente. Conclui-se, com isto, que há dificuldade de articulação das empresas conforme potencialidades internas e oportunidades do ambiente externo. Por outro lado, ao observar os limites máximos do mesmo conjunto de empresas, é possível detectar que desfrutam de atividades aceleradoras de ofertas de inovação, de modo que a utilização de mecanismos que vão da concepção das ideias à implementação e retirada do produto do mercado, identificados nos indicadores de capacidade inovativa, tende ao grau informal na média global, pois aproximam-se do índice 2, com IMAI 1,90 e 1,82, respectivamente para empresas JOIN e Gêmeas.

\subsection{Análise Individual do Comportamento das Empresas JOIN e Empresas Gêmeas}

A representação gráfica do comportamento individual das empresas, através do radar da inovação, permite melhor visualizar cada temática que agrega os indicadores da matriz de IMAI (Figura 2), a seguir.

Figura 2 - Radar da Inovação - Empresas JOIN e Gêmeas* localizadas na Região Sul da Bahia (2013-2014).

Legenda: Empresas JOIN $\multimap$ Empresas Gêmea
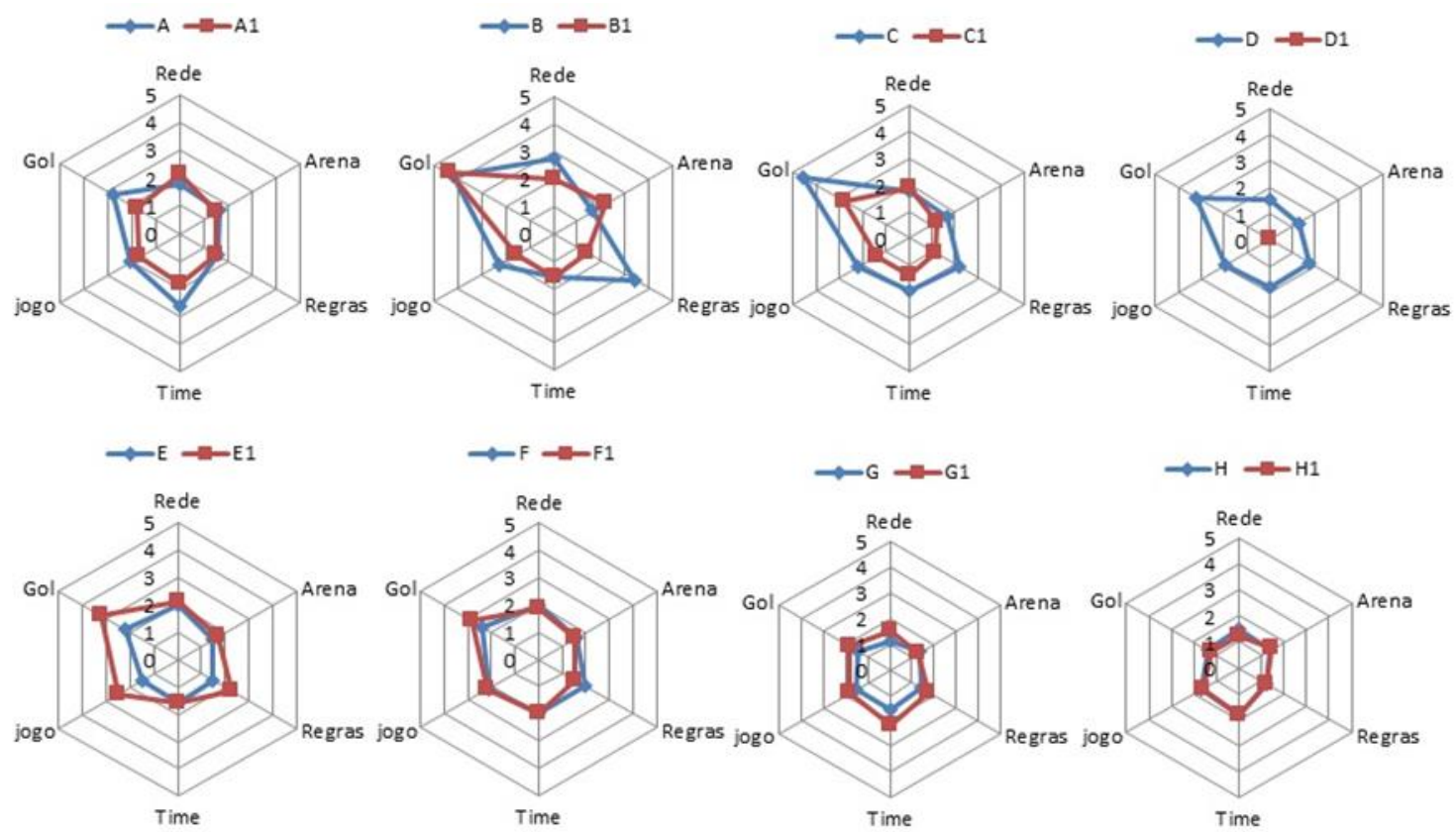

Nota: Setor de atividade A/A1: Produtos de limpeza; B/B1: Embalagens de papel; C/C1: Lanchonete e confeitaria; D: Produtos derivados do cacau e chocolate; E/E1: Produção de Argamassas; F/F1: Lanchonetes e restaurante; G/G1 Serviços gráficos; H/H1: Supermercado. (*) Empresas com perfil e setor de atividade semelhante às empresas inseridas no JOIN (2013-2014) na Região Sul da Bahia.

Fonte: Pesquisa de campo (2015). 
Como comportamento geral pode-se observar que os radares de inovação estão dispostos em uma forma que se aproxima a um losango, tendendo para maior concentração para a temática "Gol” entre as empresas A, B, C e D inseridas no JOIN e, B1, C1 e E1 entre as Gêmeas, aliado a uma distribuição mais uniforme e tímida entre as temáticas para as demais empresas. Estas empresas com maior inclinação para ofertas das inovações (Gol) em detrimento à preparação de um ambiente que possa gerar inovações melhores e de forma mais rápida (capacidades inovativas), podem acumular maior risco à atividade de inovação, pois seus esforços estão voltados mais para os fins do que para os meios pelas quais as melhorias são alcançadas.

Alinhada à visão contemporânea de que a atividade de inovação possui bases colaborativas, a concentração de esforços em oferta de inovações deve vir acompanhada de correspondente investimento em capacidades inovativas, que apoiem o desenvolvimento de novos bens e serviços de modo contínuo e consistente, pois a disponibilização de produtos com novas características por si só não representa necessariamente uma inovação. Para ser considerada uma inovação a novidade deve gerar valor adicional ao usuário e, consequentemente, à empresa, e isto é possível à medida que se adquire competências técnicas e comportamentais favoráveis ao aumento das capacidades inovativas, de modo a ir substituindo a oferta de inovações que representam baixa contribuição ao desempenho organizacional por outras de maior valor.

A empresa $\mathrm{G}$ (setor de serviços), bem como H e H1 (setor de comércio) estão mais próximas de IMAI inexistente (grau 1) do que de informal (grau 2), respectivamente com índice equivalente a 1,41, 1,49 e 1,42. Possivelmente a defasagem observada quanto ao IMAI corresponde a um comportamento natural do setor de atividade de acordo com o porte e ambiente em que estão inseridas.

O fato do IMAI tender ao nível informal na maior parte das empresas observadas, representa que o comportamento prevalecente é de que há iniciativas dos gestores em induzir e coordenar ações de inovação, porém de forma pessoal, de acordo com seus valores e afinidades. Em outras palavras, entre as atribuições da gestão incluem a busca pessoal por novas alternativas para o negócio e, esforço de encorajamento dos colaboradores para o desenvolvimento de ideias, que ocorre predominantemente por meio de incentivos verbais. Além disso, mantem-se inexistente, majoritariamente, recursos de inovação que são considerados novos pelos gestores ou de difícil apropriação, devido ao grau de maturidade da equipe (MONTANARI e PILATTI, 2010).

\section{Conclusões}

Conclui-se que as fases que marcam a transição entre os níveis de maturidade da atividade de inovação demandam recursos específicos e refletem características típicas em cada fase de evolução (Quadro 4), justificando o ritmo de desenvolvimento da gestão da inovação, pois diversos 
níveis de maturidade podem coexistir em uma organização, a depender do tamanho e da capacidade de difusão das capacidades inovativas. Desta forma, é importante investir em ferramentas de compartilhamento de valores e informações, desetorizando os recursos catalizadores de ideias, além de reforçar a cultura pró-inovação que permite evoluir para níveis de maturidade mais elevados.

Quadro 4 - Evolução, recursos e característica da transição entre os níveis de Maturidade da Atividade de Inovação

\begin{tabular}{|c|c|c|}
\hline Evolução da Maturidade & Recursos necessário & Características \\
\hline Inexistente - Informal & Tempo e iniciativa do gestor. & $\begin{array}{l}\text { O gestor centraliza a operacionalização da } \\
\text { atividade de inovação; Esforça-se em } \\
\text { inspirar os colaboradores a contribuir, mas } \\
\text { não orienta por meio de mecanismos e } \\
\text { ferramentas. }\end{array}$ \\
\hline Informal - Estruturado & $\begin{array}{l}\text { Apoio e coordenação da liderança; } \\
\text { Apoio externo; Engajamento da equipe; } \\
\text { Investimento de tempo e alocação de } \\
\text { recursos financeiros para atividade de } \\
\text { inovação. }\end{array}$ & $\begin{array}{l}\text { Adoção de mecanismos e ações estruturadas } \\
\text { para atingir os objetivos de inovação; } \\
\text { Melhora-se a comunicação interna e } \\
\text { externa; Há alocação de tempo do gestor de } \\
\text { atividades de rotina para atividades } \\
\text { estratégicas; Institui-se e difunde regras } \\
\text { para inovação e orienta a equipe para o } \\
\text { alcance dos objetivos relativo às suas } \\
\text { atividades. }\end{array}$ \\
\hline Estruturado - sistematizado & $\begin{array}{l}\text { Maturidade de estruturação das } \\
\text { atividades; experiência adquirida na } \\
\text { estruturação e transformação de } \\
\text { conhecimento tácito em explícito. }\end{array}$ & $\begin{array}{l}\text { Há diminuição significativa do risco da } \\
\text { atividade inovativa; Rapidez na geração de } \\
\text { inovação; implementação de inovações com } \\
\text { maior valor agregado e com maior grau de } \\
\text { novidade. }\end{array}$ \\
\hline Sistematizado - Otimizando & $\begin{array}{l}\text { Maturidade da Sistematização e tempo } \\
\text { para que as práticas sistematizadas } \\
\text { gerem competências internas capazes } \\
\text { de gerir um processo contínuo de } \\
\text { revisão e melhorias. }\end{array}$ & $\begin{array}{l}\text { know-hall na área de atuação; Capacidade } \\
\text { de difundir sua tecnologia de gestão em } \\
\text { consultorias na área de atuação; Capacidade } \\
\text { de desenvolver Spin-off; Inspira soluções. }\end{array}$ \\
\hline
\end{tabular}

Fonte: Autoria própria (2015).

Argumenta-se que é relevante a Maturidade da Atividade de Inovação tendendo ao nível informal, identificada nas MPEs da Região Sul da Bahia, pois pondera-se a especificidade dos indicadores, pouco comuns em empresas dos ramos estudados. O nível informal representa o primeiro estágio de gestão da inovação, no qual, ainda assimila certo conservadorismo e consiste no nível de maior dificuldade de superação, em contrapartida, o nível estruturado (que utiliza ainda que de forma parcial procedimentos padronizados e instrumentos formais de controle) é um nível de alta relevância no universo de empresas identificadas, principalmente quando não passaram por nenhuma intervenção especializada na área de inovação, pois o nível otimizando consiste num estágio ideal que requer amadurecimento na gestão da inovação.

Destaca-se que para isolar o efeito dos resultados do projeto JOIN sobre suas contratantes, os dados desta pesquisa foram coletados antes da sua implementação nas empresas, no entanto, apesar do contrato duas empresas não chegaram a executá-lo $(G$ - serviços gráficos e $H$ supermercado). De modo análogo, os resultados da pesquisa revelaram um comportamento menos competitivo para estes dois setores de atividade: Estas empresas são as únicas incluídas no grupo 
"Empresas JOIN" que tendem ao nível inexistente de Maturidade da Atividade de Inovação. Quanto às respectivas Gêmeas, tem-se que a empresa $\mathrm{H} 1$ também tende a IMAI inexistente, enquanto a empresa de serviços gráficos (G1) anunciou descontinuidade de suas atividades em 2015, apesar de sustentar um índice de maturidade tendendo a informal. Evidencia-se, com isto, que a maturidade em termos de inovação varia de acordo com o setor de atividade, somando-se à inclinação para projetos de inovação.

Como elementos de distinção entre os dois grupos de empresas estudados, destaca-se que além da média das empresas JOIN terem apresentado maturidade em inovação superior às empresas Gêmeas, após a efetiva implementação da tecnologia JOIN, as contratantes possuirão subsídios para avançar para os próximos níveis de maturidade. Assim, constitui recomendação de pesquisas futuras a investigação da relação entre efetiva implementação e eficácia do projeto, contribuindo em última instancia na avaliação da política pública que o JOIN está ancorado. Além disto, recomenda-se ampliação da amostra e concentração do estudo em um setor de atividade para verificar peculiaridades das empresas inseridas em um mesmo setor.

A quantidade reduzida de empresas com as características de Gêmeas, aliado à indisponibilidade de agenda dos gestores para fornecer os dados da pesquisa, constituíram limitações à operacionalização desta pesquisa, resultando na lacuna de uma empresa devido à falta de voluntários que representasse o grupo de empresas Gêmeas relativo ao setor de produtos derivados do cacau e chocolate. Uma perda especialmente significativa, devido a contribuição do setor para o desenvolvimento regional do Sul da Bahia, já que é símbolo da área de estudo, conhecida como região cacaueira.

\begin{abstract}
Built in the logic of public policy increase of competitiveness and business development aimed at raising the quality and perceived value of domestic products and services, project support innovation activity has been structured and brought to market in recent years, making -If important to identify the features and innovation performance of the companies involved. Therefore, this article is concerned with delineating the behavior of companies, located in the South of Bahia, on the management of innovation from an experimental method that included application of the indicators of innovation JOIN Matrix (2013) and conducting a comparison between an experimental group, characterized by contractors innovation project - JOIN and a group of companies that have similar characteristics, but does not participate in the project - called Twin enterprises (control group). The results indicate that the JOIN companies have superior performance at maturity in innovation activity, confirming the hypothesis that companies adhere to innovation projects tend to have different characteristics, which give them greater powers to innovate.
\end{abstract}

Key-words: Diagnosis; Maturity; Innovation; Game of innovation.

\title{
Referências
}


BRANDÃO, L. A; DRUMOND, C. E. I. Políticas pró-inovação: uma análise da política industrial nos oito anos do governo Lula. Economia \& Tecnologia,v. 8, n. 2, p. 20-40, 2012. DOI: http://dx.doi.org/10.5380/ret.v8i2.28183. crossref

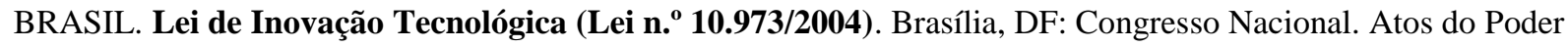
Legislativo, DOU, n. ${ }^{\circ} 232$ de 03.12.2004.

BRASIL. Lei do Bem (Lei n. ${ }^{\mathbf{1} 11.196 / 2005)}$. Brasília, DF: Congresso Nacional. Atos do Poder Legislativo, DOU, n. ${ }^{\circ}$ 232 de 03.12.2004.

CHESBROUGH, H.W. Open Innovation: researching a new paradigm. Oxford University Press, 2006.

FINEP. Chamada pública MCT/FINEP/Pró-Inova 11/2010: Núcleos de Apoio à Gestão da Inovação. 2011. Disponível em: <http://www.finep.gov.br//fundos_setoriais/acao_transversal/resultados/Chamada\%20NAGI\%20\%20Resultado\%20Final.pdf >. Acesso em: 01 de nov. 2014.

JOIN. Portal Jogo da Inovação. 2013. Disponível em <www.jogodainovacao.com.br>. Acesso em: 27 Fev. 2015.

MANUAL DE OSLO. OECD-EC. Diretrizes para coleta e interpretação de dados sobre inovação (1997). Tradução de Flávia Gouveia. 3. ed. Rio de Janeiro: Arti/Finep, 2005.

McAFEE, A. Empresas 2.0: a força das mídias colaborativas para superar grandes desafios empresariais. Rio de Janeiro, 2010.

MONTANARI, R. L; PILATT, L.A. As Influências do Grau de Maturidade da Equipe nos Processos de Inovação Tecnológica e Construção do Conhecimento Organizacional. Revista de Economia Política de las Tecnologías de la Información y Comunicación. Vol XII, n. 2, Mayo-Agosto/2010. Disponível em<

http://www.seer.ufs.br/index.php/eptic/article/viewFile/74/48>. Acesso em: 10 nov. 2014.

NONAKA, I; TAKEUCHI, H. Gestão do Conhecimento. Porto Alegre: Bookman, 2008.

SCHUMPETER, J. A. Capitalismo, socialismo e democracia. [S.1.: s.n.], 1942.

TIGRE, P. B. Gestão da inovação: a economia da tecnologia no Brasil. Rio de Janeiro: Campus, 2006. 282p.

Sistema FIEB. JOIN é replicado em Pernambuco. Disponível em <http://www.fieb .org.br/apoio_a_industria/Noticia/2393/join-e-replicado-em-pernambuco.aspx>. Acesso em: 01 Dez. 2014.

VIANNA, M. et al. Design thinking: Inovação em negócios. Rio de Janeiro: MJV Press, 2012.

ZEN, A. C. et al. O Papel do Governo no Apoio à Gestão de Inovação das Empresas: um estudo exploratório do Programa de Núcleos de Apoio à Gestão de Inovação. XXIV Seminários Nacional de Parques Tecnológicos e Incubadoras de Empresas. 2014.

\section{Dados dos autores:}

Nome completo: Leidiane Alcântara Brandão

Filiação institucional: UESC - Universidade Estadual de Santa Cruz (Bahia)

Departamento: Ciências Ambientais

Endereço completo para correspondência (bairro, cidade, estado, país e CEP): Mestrado em Desenvolvimento Regional e Meio Ambiente - Campus Soane Nazaré de Andrade - km 16 Rodovia Ilhéus-Itabuna, Salobrinho - Ilheus, BA - Brasil CEP 45662-000.

e-mail: brandao.leidiane@gmail.com 
Nome completo: Andréa da Silva Gomes

Filiação institucional: UESC - Universidade Estadual de Santa Cruz (Bahia)

Departamento: Ciências Econômicas

Função ou cargo ocupado: Professora

Endereço completo para correspondência (bairro, cidade, estado, país e CEP): Campus Soane Nazaré de Andrade - km 16 Rodovia Ilhéus-Itabuna, Salobrinho - Ilheus, BA - Brasil CEP 45662000.

e-mail: andreauesc@gmail.com

Nome completo: Gesil Sampaio Amarante Segundo

Filiação institucional: UESC - Universidade Estadual de Santa Cruz (Bahia)

Departamento: DCET - Departamento de Ciências Exatas e Tecnológicas

Função ou cargo ocupado: Professor / Coordenador do Núcleo de Inovação Tecnológica da UESC

Endereço completo para correspondência (bairro, cidade, estado, país e CEP): Campus Soane Nazaré de Andrade - km 16 Rodovia Ilhéus-Itabuna, Salobrinho - Ilheus, BA - Brasil CEP 45662000.

e-mail: gesil.amarante@gmail.com

Submetido em: 23-03-2015

Aceito em: 14-09-2015 